\title{
Malaria treatment, compliance and cure in Gandhinagar, Gujarat
}

\author{
Mamta Dattani ${ }^{1}$, George Kurien ${ }^{2}$ \\ ${ }^{1}$ District Panchayat, Gandhinagar, Gujarat, India; mldattani@yahoo.co.in \\ ${ }^{2}$ National Vector Borne Disease Control Programme, Commissionerate of Health, Gandhinagar, Gujarat, India
}

Received 31 October 2013; revised 28 November 2013; accepted 21 December 2013

Copyright (C) 2014 Mamta Dattani, George Kurien. This is an open access article distributed under the Creative Commons Attribution License, which permits unrestricted use, distribution, and reproduction in any medium, provided the original work is properly cited. In accordance of the Creative Commons Attribution License all Copyrights (C) 2014 are reserved for SCIRP and the owner of the intellectual property Mamta Dattani, George Kurien. All Copyright (C) 2014 are guarded by law and by SCIRP as a guardian.

\section{ABSTRACT}

Early diagnosis and complete treatment are the most important components of the malaria control program, which have a direct bearing on prevention of mortality and reduction in the morbidity, therefore, follow-up of the patient treatment is an important aspect of monitoring disease in the community. Aims: To assess treatment compliance among confirmed malaria cases in Gandhinagar District and to determine the proportion of cure rate with complete treatment as per the national drug policy. Study Variables: Exploratory-rural and urban areas; outcomecompliance, cure rate. Analysis: Percentage, proportions. Results: Compliance among 192 subjects under the study conducted in 2008 i.e. confirmed that malaria cases were $88 \%$ even after the introduction of 14 day therapy for "Plasmodium vivax" cases, while $100 \%$ cure rate was observed on the follow-up till the 28th day among " $P$. vivax" and " $P$. falciparum" cases which helped in reducing malaria incidence in the rural area by $50 \%$ as compared to the preceding year of the study. Conclusion: Follow-up of the treatment given to malaria patients has enhanced compliance and cure rate, which in turn contributed towards interruption of transmission. Therefore, a better cure rate through the administration of effective antimalarial drugs as per the drug policy in combination with other containment measures is the right strategy to keep morbidity and mortality due to malaria under effective control.

\section{KEYWORDS}

Malaria; Compliance; Treatment

\section{INTRODUCTION}

In Gujarat, Vector Borne Diseases viz. Malaria, Dengue and Chikungunya (since 2006) are major public health problems in the community. Malaria continues to be a major vector borne disease and accounts for a major part of the morbidity, though there has been a significant and constant decline in total incidence of malaria in the state since 2004 [1]. All the vector borne diseases have been brought under the ambit of National Vector Borne Disease Control Programme (NVBDCP) since 2004 to implement the integrated approach for prevention and control as well as to have synergistic approach which has become possible with the implementation of National Rural Health Mission [2]. Malaria in Gujarat state is unstable and contributes to only $2.9 \%$ incidence of malaria reported from India (2009). Anopheles culicifacies is the main vector of rural and peri-urban malaria. Vector control is based on Indoor Residual Spray with synthetic pyrethroid (Alphacypermethrine) as well as Malathion and anti-larval operation (temephos, bio-larvicide and larvivorous fishes). District Malaria Officer heads malaria control programme at district level. In villages of south and central Gujarat, chloroquine resistant $P$. falciparum (CRPF) has been reported [3]. Similarly, CRPF was also reported in labors working at construction site, agriculture field and urban area in different districts of Gujarat [4]. Since 1990s outbreaks/epidemics of malaria occurred in many parts of the state [5], genotype investigation of " $P$. vivax" cases had confirmed two true chloroquine treatment failures in Anand District [6]. For sustainable malaria control, besides the availability of appropriate tools, monitoring of treated malaria patients is an important aspect of malaria control programs. The modification in the drug policy has necessitated the surveillance mechanism to follow up the treatment provided for malaria cases to ensure treatment compliance as well as to 
have an idea about cure rate and to detect early or late treatment failure. The present study was undertaken in 2008-2009 to assess the level of compliance and susceptibility status of parasite against drug used (Chloroquine and Primaquine).

\section{PATIENTS AND METHODS}

\subsection{Study Area}

The study was carried out in Gandhinagar district having a total population of 15.47 lakh. There are four talukas and 294 villages which are having a very good network of health facilities which comprises 171 sub centers, 24 Primary Health Centers and 7 Community Health Centers. Agriculture, animal husbandry and dairy farming are the main occupation in rural area. The local population is stable and short term migrant laborers are employed. The 3 talukas of district receives irrigation by Sardar Sarovar Narmada canal net work.

\subsection{Enrolment, Treatment and Follow Up}

All the PHCs (24), CHCs [7) and hospitals [2] of four talukas were included in study. Multi-purpose health workers (MPHWs) visit each family once every fortnight. During the family visits, the MPHW takes blood smears of the febrile patients on glass slides and administers treatment (chloroquine $25 \mathrm{mg} / \mathrm{kg}$ body wt) for suspected malaria infection. Additionally there are Fever Treatment Depots run by volunteers who also make blood slides and administer treatment. All blood smears collected by ACD and PCD were deheamoglobinised, stained with JSB stain and examined by Laboratory Technicians at the PHC. All confirmed malaria cases were provided treatment according to National Drug policy i.e. Chloroquine and Primaquine to " $P$. vivax" and " $P$. falciparum" [7]. Each microscopically confirmed malaria case was followed up by MPHW as per the modified drug policy on day 3, 7, 14 and 28 post-treatments. On each day of follow up blood smear was taken for microscopic examination. A treatment follow up card was developed in the local language (English version annex-1), which was used by the concerned Multi Purpose Health Supervisor of the PHC to collect the information regarding treatment administrated and the follow up slides collected from the patients included in the study. The information collected from the field as per the prescribed treatment card was verified by the Laboratory Technician and Medical Officers of the PHCs to have vital inputs and feedback regarding treatment compliance and treatment failure.

\section{RESULTS}

During 2008, out of 236,327 blood smears of fever patients screened for malaria 500 (88.96\%) and 62 (11.03\%) were positive for " $P$. vivax" and " $P$. falciparum" respectively. 409 (72.77\%) of the total malaria cases detected in the district were from the urban area while 153 (27.2\%) cases were from the rural area. Gandhinagar taluka contributed the maximum numbers of malaria cases (79.89\%) in the district out of the four talukas. Males constituted $63.87 \%$ of the total cases detected [8]. As regards age group wise classification maximum cases (81.13\%) were in the age group of $>14$ years, while $1-4$ and 5 - 14 years of age groups constituted $4.98 \%$ and $13.89 \%$ of the cases reported respectively. No infant was detected as malaria positive (Table 1 ).

Out of 562 malaria cases detected in the district, 192 cases (34\%) were covered under the study and the treatment cards were also filled up. Looking to the feasibility of collecting the information and availability of staff to undertake the work more emphasis was given to the rural areas and all the subjects included in the study were followed up, while in urban areas only $9.53 \%$ of the cases could be followed up which indicates the constraints in tracking the cases in an urban settings where the infrastructural facilities are deficient as compared to the rural area.

Of the 192 confirmed malaria cases who were included in the study, 173 were " $P$. vivax" (90.10\%) and 19 were " $P$. falciparum" (9.10\%). These cases were spread over 57 villages of 22 PHCs and 3 urban areas. The subjects included in the study which were followed up comprises of $59.89 \%$ of male patients while $40.11 \%$ were female patients. Majority of the cases (73.95\%) were in the age group of 15 years and above, while $4.98 \%, 6.77 \%$ and $14.58 \%$ were in the age group of $1-4$, 5 - 8 and 9 - 14 years respectively (Table 2). Infants and pregnant women were not included among the subjects of the study.

Findings of the study as shown in Table 3 reveals a compliance of $88.02 \%$ as 169 patients completed their

Table 1. Malaria cases detected in Gandhinagar District during 2008.

\begin{tabular}{|c|c|c|c|c|c|c|c|c|c|c|}
\hline \multirow{2}{*}{ Age group (years) } & \multicolumn{5}{|c|}{ Male } & \multicolumn{5}{|c|}{ Female } \\
\hline & Cases & Pvivax & Pfalci parum & Mix & Pfalci parum \% & Cases & Pvivax & Pfalci parum & Mix & Pfalci parum \% \\
\hline $0-1$ & 0 & 0 & 0 & 0 & 0 & 0 & 0 & 0 & 0 & 0 \\
\hline $5-14$ & 44 & 36 & 8 & 0 & 18.18 & 34 & 27 & 7 & 0 & 20.59 \\
\hline$>14$ & 297 & 267 & 30 & 0 & 10.10 & 159 & 143 & 16 & 0 & 10.06 \\
\hline All age groups & 359 & 320 & 39 & 0 & 10.86 & 203 & 180 & 23 & 0 & 11.33 \\
\hline
\end{tabular}


Tab le 2. Malaria cases covered under the current study.

\begin{tabular}{ccccccccccc}
\hline \multirow{2}{*}{ Age group (years) } & \multicolumn{9}{c}{ Male } & \multicolumn{5}{c}{ Female } \\
\cline { 2 - 11 } & Cases & Pvivax & Pfalci parum & Mix & Pfalci parum \% & Cases & Pvivax & Pfalci parum & Mix & Pfalci parum \% \\
\hline $0-1$ & 0 & 0 & 0 & 0 & 0 & 0 & 0 & 0 & 0 & 0 \\
$1-4$ & 6 & 6 & 0 & 0 & 0 & 3 & 3 & 0 & 0 & 0 \\
$5-8$ & 8 & 5 & 3 & 0 & 37.5 & 5 & 4 & 1 & 0 & 20 \\
$9-14$ & 18 & 17 & 1 & 0 & 5.56 & 10 & 9 & 1 & 0 & 10 \\
15 \& above & 79 & 72 & 7 & 0 & 8.86 & 63 & 57 & 6 & 0 & 9.52 \\
All age groups & 111 & 100 & 11 & 0 & 9.90 & 81 & 73 & 8 & 0 & 9.87 \\
\hline
\end{tabular}

Tab le 3. Treatment compliance and results of follow up.

\begin{tabular}{|c|c|c|c|c|c|c|c|c|}
\hline \multirow{2}{*}{ Age group (years) } & \multirow{2}{*}{ Cases studied } & \multirow{2}{*}{ Treatment completed } & \multicolumn{6}{|c|}{ Follow up smears of cases studied } \\
\hline & & & 3rd day & 7th day & 14th day & 28th day & Total & Results \\
\hline $0-1$ & 0 & 0 & 0 & 0 & 0 & 0 & 0 & Negative \\
\hline $1-4$ & 9 & 7 & 3 & 5 & 6 & 6 & 21 & Negative \\
\hline $5-8$ & 13 & 10 & 9 & 8 & 10 & 7 & 34 & Negative \\
\hline $9-14$ & 28 & 26 & 25 & 23 & 25 & 24 & 97 & Negative \\
\hline 15 \& above & 142 & 126 & 109 & 109 & 108 & 93 & 419 & Negative \\
\hline All age groups & 192 & 169 & 147 & 145 & 149 & 130 & 571 & Negative \\
\hline
\end{tabular}

treatment out of 192 cases followed up. Non compliance was less in case of " $P$. vivax" (8.8\%) as compared to " $P$. falciparum" (24\%). Non compliance was found to be less in rural areas $(5.88 \%)$ as compared to urban areas (35.89\%). The comparatively higher rate of non compliance among the falciparum cases studied were due to the high proportion of non compliance in urban areas as $42 \%$ of falciparum cases which have not completed the treatment were from the urban areas.

Out of the 169 subjects covered under the study 147 were followed up, of which only 130 were followed up on all the four days. Blood smears from 147 patients who were followed up were collected on the 3rd day while blood smears of 145 and 149 were collected on 7th and 14th day. 130 patients were followed up on the 28th day. Microscopic examination of follow up smears did not revealed the presence of malaria parasite. Contact smears from 668 family members of the malaria cases were collected, but microscopic examination revealed the same to be negative for malaria parasite.

\section{DISCUSSION}

As the health personnel were motivated and sensitized to participate in this study there was no problem in the field to collect the information and collecting follow up smears through these workers. However there was some reluctance on the part of patients to give follow up smears at frequent intervals. But most of them could be convinced to co-operate in the study. Better compliance is also achieved because of the active involvement of the staff working under the Primary Health Care delivery system.

The most important observation is that none of the follow up smears was positive for malaria parasite, indicating a $100 \%$ cure rate or rather no treatment failure in this district among the studied subjects. This substantiates the fact that the treatment being imparted as per the drug policy is quite effective to cure the patients clinically as well as parasitologically. The administration of Primaquine is one of the reasons for achieving the desired impact on transmission. The significant decline of malaria incidence (52\% reduction) in the rural areas of Gandhinagar district in 2008 as compared to 2007 can be attributed to the effective mechanism for providing complete treatment to malaria patients as the villages covered under Indoor Residual Spray were only 16. This reveals that deployment of effective anti malarial drugs either alone or in combination with other containment measures can reduce morbidity and mortality due to malaria $[9,10]$. The follow up smears were cross checked in the district laboratory to confirm the results of the PHCs.

The Major contributory factors for non compliance in treatment were migration. Detailed analysis of these patients have revealed that $4.68 \%$ has left the place to an area outside the limits of district while $3.12 \%$ could not be traced.1.04\% were pregnant women and $1.04 \%$ had refused to take treatment. Very few patients (2.08\%) reported mild reactions such as itching, vomiting etc. due to the intake of drug and therefore treatment were discontinued in such patients.

The study has brought out the important fact that treatment of malaria cases has to be a directly observed one to improve compliance. The other important finding is that if due care is taken to give directly observed treatment the compliance rate can be as good as $88.02 \%$, which is quite satisfactory in a public health programme. However compliance in the urban areas requires improvement which will require sensitization of the staff involved in surveillance as well as those who provide 
treatment. More efforts on the part of the surveillance agencies to line list the cases properly and to follow up the same through the health supervisors will be very vital in the urban areas. The study also reveals that the drug regimen as per the drug policy of NVBDCP is effective. Chloroquine was found to be effective for the treatment of "P. falciparum" cases (Directorate of NVBDCP has changed the drug policy and now " $P$. falciparum" cases are to be treated with ACT combination therapy).

\section{CONCLUSION}

This study clearly shows that adherence to the national drug policy along with better compliance can play a vital role in interrupting transmission of malaria as well as reducing the parasitic reservoir in the community. This gives a direction regarding the importance of directly observed treatment and also the need to have a systematic follow-up mechanism through the surveillance machinery particularly for low endemic areas (districts like Gandhinagar) where case detection and complete treatment are of greater values to achieve the objectives of the program. A better cure rate through the administration of effective antimalarial drugs as per the drug policy in combination with other containment measures is the right strategy to keep morbidity and mortality due to malaria under effective control.

\section{ACKNOWLEDGEMENTS}

The authors dedicate this paper to late Dr. P. B. Prajapati who was Joint Director, NVBDCP Gujarat. He took keen interest and played a leading role in initiating this study. He has been the guiding force throughout the period of study and has provided valuable in puts in terms of technical knowhow, administrative support and continuous motivation. The commitment and sincerity shown by the NVBDCP team at the district head quarter and also Multi Purpose Health Workers, Multi Purpose Health Supervisors, Medical Officers, Laboratory Technicians and AYUSH Medical Officers of Primary Health Centers and Block Health Officers of Block Health Offices is duly acknowledged. The support received from state Health Society-NVBDCP and District Health Society-NVBDCP in terms of technical and financial inputs was of immense help. The efforts made by Shri Nayak Bankesh and Shri Vasavda Perry, Laboratory Technicians of NVBDCP branch, Gandhinagar district for compilation and analysis of the data were quite useful for this study. The cooperation received from the patients was the key aspect for the successful completion of the study and therefore the same is greatly acknowledged.

\section{REFERENCES}

[1] “Annual Report,” National Vector Bone Disease Control Programme, Gujarat, 2009.

[2] "Strategic Action Plan for Malaria Control in India, 20072012,” Directorate of National Vector Borne Disease Control Programme, Delhi.

[3] R. C. Sharma and V. P. Sharma, "Epidemiological Implications of Population Migration-Part-II: Evidence of Chloroquine Resistant Plasmodium Falciparum Malaria in Kheda District of Gujarat," Indian Journal of Malariology, Vol. 25, No. 2, 1988, pp. 117-118.

[4] H. C. Srivastav, C. S. Pant, R. S. Yadav and P. B. Prajapati, "Malaria Transmission in Seasonal Migrants in South Gujarat: Obstacle to Malaria Control,” 2009.

[5] H. C. Shrivastav, R. M. Bhatt, R. S. Yadav and V. P. Sharma, "Malaria Outbreak in Tribal Areas of Gujarat State-India," South East Asian Journal of Tropical Medicine and Public Health, Vol. 31, No. 2, 2000, pp. 219223.

[6] H. C. Srivastav, R. S. Yadav, H. Joshi, N. Valecha, P. K. Mallik, S. K. Prajapati and A. P. Dash, "Therapeutic Responses of 'Plasmodium vivax' and ' $P$. falciparum' to Chloroquine in an Area of Western India Where $P$. vivax Predominates," Annals of Tropical Medicine and Parasitology, Vol . 102, No. 6, 2008, pp. 471-480. http://dx.doi.org/10.1179/136485908X311759

[7] “National Anti Malaria Drug Policy,” National Vector Borne Disease Control Programme, 2009.

[8] "National Vector Borne Disease Control Programme," Gandhinagar District, Annual Report, 2009.

[9] Tijitra et al., "Efficacy of Chloroquine, Chloroquine plus Sulphadoxine-Pyrimethamine and Amodiaquine for Treatment of Vivax Malaria in Bangka Island, Indonesia: A Randomized Trial,” Medical Journal of Indonesia, 1996.

[10] N. J. White, “The Role of Anti-Malarial Drugs in Eliminating Malaria,” Malaria Journal, Vol. 7, Suppl. 1, 2008, p. S8. http://dx.doi.org/10.1186/1475-2875-7-S1-S8 